\title{
LIMITER BIAS EXPERIMENTS IN DIII-D
}

\author{
by \\ M. SHIMADA, ${ }^{*}$ A. OZAKI, ${ }^{\dagger}$ \\ P. PETERSEN, P. RIEDY, B. BURLEY, \\ T. PETRIE, G. JANESCHITZ ${ }^{\ddagger}$ M.A. MAHDAVI \\ *Japan Atomic Energy Research Institute \\ †Toshiba Corporation \\ $\ddagger_{\text {Max-Planck Institute for Plasma Physics }}$
}

This is a preprint of a paper to be presented at the 9th International Conference on Plasma Surface Interactions, May 21-25, 1990, Bournemouth, United Kingdom and to be printed in the Proceedings.

\author{
Work supported by \\ Department of Energy \\ Contract DE-AC03-89ER51114
}
GENERAL ATOMICS PROJECT 3466
NOVEMBER 1990

\section{GEMERAL ATOMICS}




\begin{abstract}
In DIII-D, $\mathrm{H}_{\alpha}$ emission was reduced by a factor of $2-3$ both at the limiter and at the inner wall by negative biasing $\left(V_{\text {lim }} \approx-300 \mathrm{~V}\right)$. In these discharges, the lineaverage electron densities were approximately the same with and without limiter bias, which suggests that $\tau_{\mathrm{p}}$ was improved by negative bias. Edge Bremsstrahlung emission signals were reduced by a factor of two. Repetitive bursts were observed in $\mathrm{H}_{\alpha}$ signals and edge Bremsstrahlung signals. Impurity levels increased with limiter bias, which induced major disruptions. No improvement of $\tau_{\mathrm{E}}$ was observed. These experimental data suggest that particle confinement characteristics of limiter biased discharges are similar to those with divertor $\mathrm{H}$-mode.
\end{abstract}




\section{INTRODUCTION}

Understanding of transport and improvement of confinement are very important subjects in thermonuclear fusion research. Recent H-mode experiments in DIII-D [1] and JFT-2M [2] show that radial electric field just inside the separatrix becomes more negative after an L-H transition. In DIII low-recycling divertor, floating potential near the separatrix became more negative with improved confinements [3]. Limiter biasing experiments in MACROTOR [4], PBX [5], TEXT [6], and TEXTOR [7] demonstrated that by applying negative electric potential at the tokamak boundary, substantial improvement or change is obtained in particle confinement and recycling. Limiter biasing experiments in PBX with neutral beam heating show a modest improvement of energy confinement [5]. Recent CCT electron injection experiments [8] demonstrated formation of transport barrier by current injection. All these experiments indicate that edge radial electric field plays a very important role in confinement.

The objective of this paper is to discuss limiter bias experiments in DIII-D with ohmically heating and neutral beam heating to investigate effects of externally applied edge radial electric field in a large tokamak. 


\section{EXPERIMENT}

The DIII-D device, its heating system, and its diagnostics are described elsewhere $[1,9]$. Electric potential was applied to a carbon limiter by using a circuit shown in Fig. 1. This limiter on the outer midplane was radially inserted by $10 \mathrm{~cm}$ from the normal position to separate the limiter-defined surface from the nonbiased material structure.

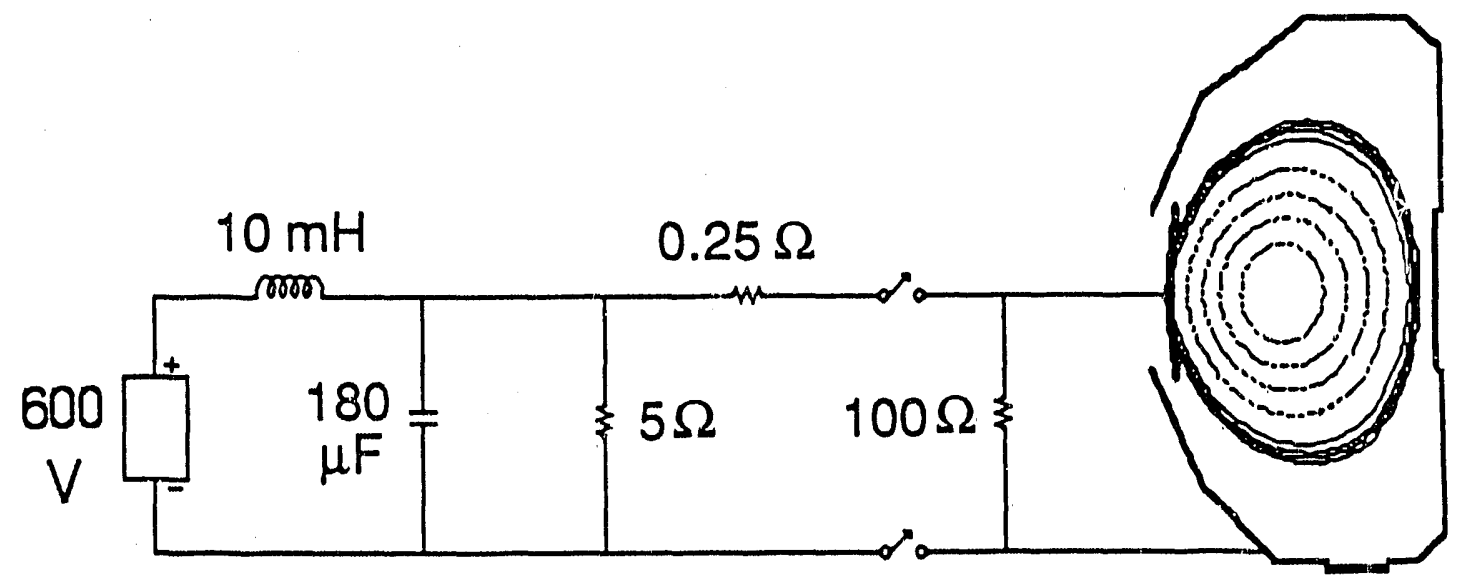

\section{Circuit Diagram}

Fig. 1. Circuit diagram for the limiter bias experiments. Equilibrium shape and the limiter are also shown. 
Figure 2 shows an ohmically heated deuterium discharge with positive bias. The toroidal field was $1.8 \mathrm{~T}$, plasma current was $800 \mathrm{kA}$. The limiter potential was ramped up slowly to observe the discharge behavior with small voltages. Edge Bremsstrahlung signals $(r / a \approx 0.8)$ and radiation loss power shows very interesting characteristics. These signals decrease as the limiter voltage is ramped up, showing minimum at $\approx 100 \mathrm{~V}$. Above $100 \mathrm{~V}$, they start to increase up to the limiter voltage of $\approx 200 \mathrm{~V}$. No significant change was observed in electron density, central Bremsstrahlung emission, $\mathrm{H}_{\alpha}$ emission at the limiter and the wall with positive bias.

Figure 3 shows an ohmically heated discharge with negative bias. For small limiter voltages, limiter $\mathrm{H}_{\alpha}$ increases from prebias values, whereas wall $\mathrm{H}_{\alpha}$, edge Bremsstrahlung, and radiation power decreases. As the limiter voltage becomes more strongly negative, $\mathrm{H}_{\alpha}$ signals at the limiter start to decrease, and $\mathrm{H}_{\alpha}$ signals both at the limiter and at the inner wall were reduced by a factor of $2-3$ at $\mathrm{V}_{\mathrm{lim}} \approx-300 \mathrm{~V}$. The radiation loss increases very rapidly up to $100 \%$ of input power, which made the discharge very unstable. In these discharges, the line-average electron densities were approximately the same with and without liniter bias, which suggests that $\tau_{p}$ was improved by negative bias. Edge Bremsstrahlung emission signals were reduced by a factor of two, suggesting that edge electron density was reduced with limiter bias. Steeper density gradient and smaller particle flux again suggests that particle confinement is improved with negative bias. Repetitive bursts were observed in $\mathrm{H}_{\alpha}$ signals and edge Bremsstrahlung signals. No improvement of $\tau_{\mathrm{E}}$ was observed, which may be due to excessive radiation loss. These experimental data suggest that particle confinement characteristics and edge plasma properties of limiter biased discharges are similar to those with divertor $\mathrm{H}$-mode.

Similar experiments were carried out to investigate effects of limiter biasing with neutral beam heating. Figure 4 shows a discharge with neutral beam heating power of 1.6 MW and $\mathrm{p} \epsilon \mathrm{ck}$ limiter voltage of $-250 \mathrm{~V}$. The working gas was deuterium and beam species was hydrogen. The toroidal field was $1.8 \mathrm{~T}$ and plasma current was $800 \mathrm{kA}$. 


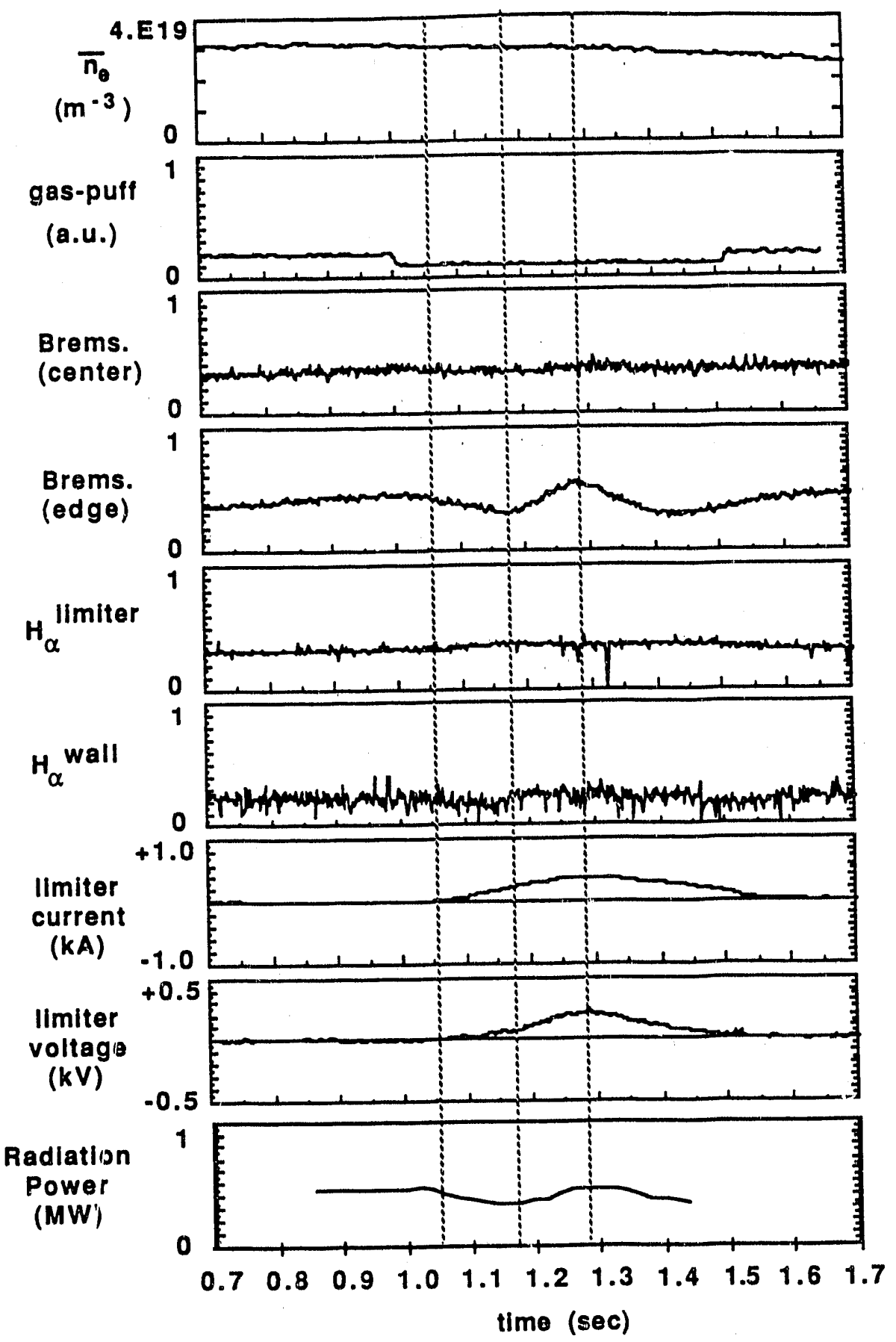

Fig. 2. Positive divertor bias with ohmic heating. 


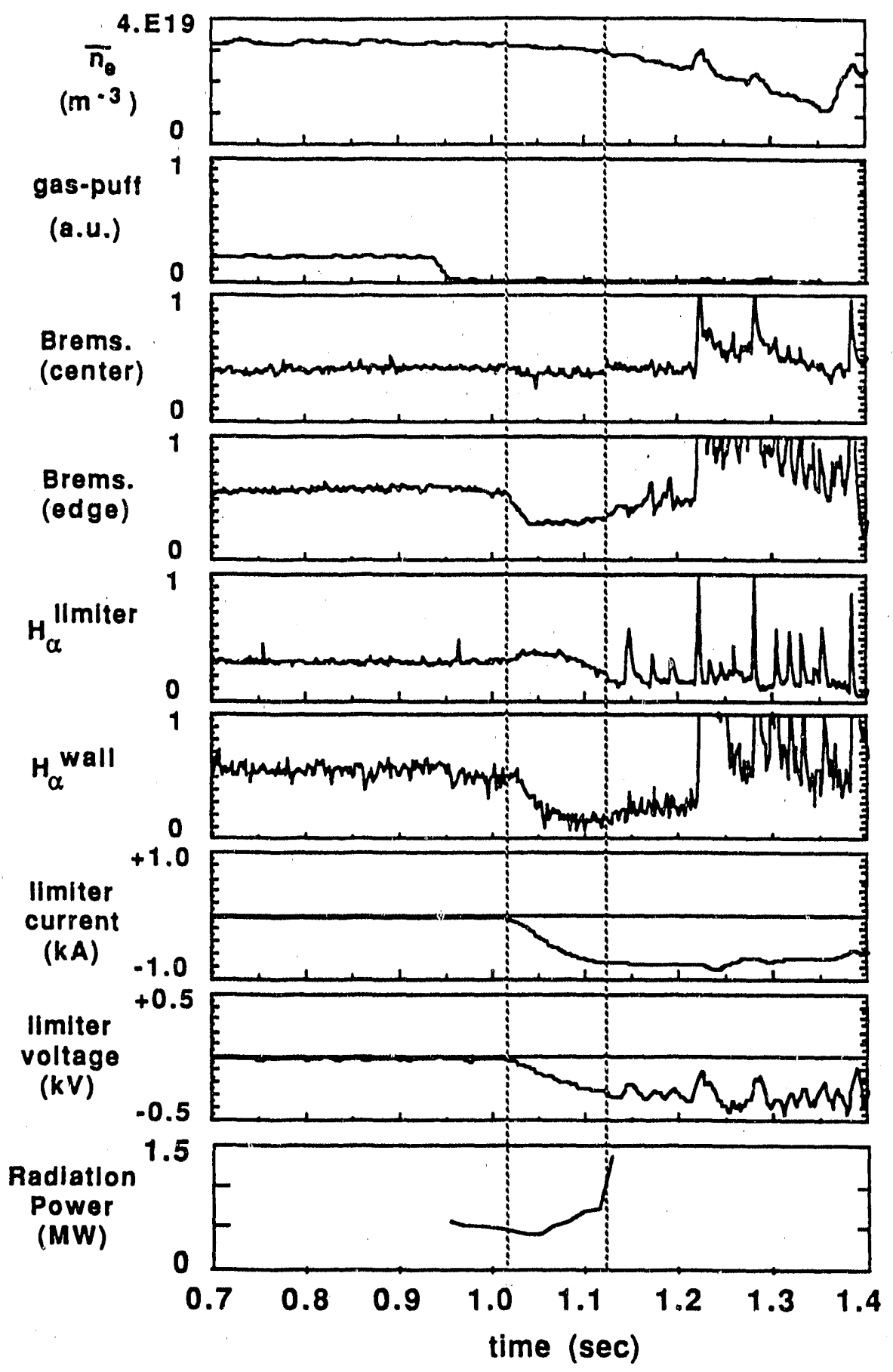

Fig. 3. Negative divertor bias with ohmic heating. 


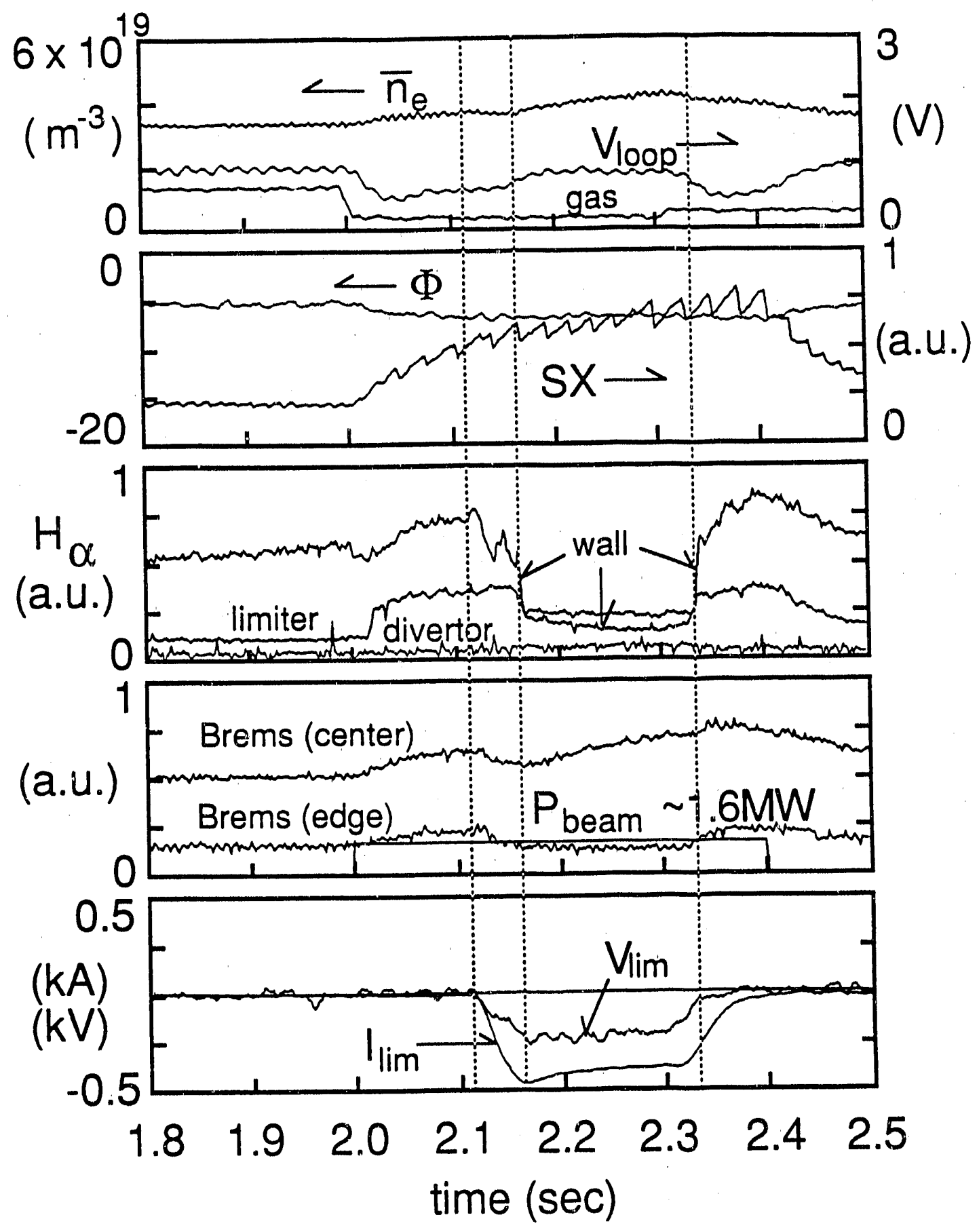

Fig. 4. Negative divertor bias with 1.6 MW neutral beam heating. 
With small limiter voltages $\left(0>V_{\text {lim }}>-200 \mathrm{~V}\right)$, the $H_{\alpha}$ emission from the wall decreased from the prebias levels. However, the $\mathrm{H}_{\alpha}$ emission from the limiter showed a slight increase. With stronger limiter voltages $\left(\mathrm{V}_{\text {lim }}<-200 \mathrm{~V}\right)$, both the limiter $\mathrm{H}_{\alpha}$ and wall $\mathrm{H}_{\alpha}$ emissions went down precipitously. The electron density increased at this moment, which shows that particle confinement is improved. Line intensities of carbon, oxygen, and nickel increased. Improvement in energy confinement was not observed.

iNeutral beam power was increased to investigate discharge characteristics with higher heating power. In this experiment, it was difficult to reproduce transition-like characteristics observed in Fig. 4 even with heating power similar to Fig. 4. The reason for the change in discharge behavior is not clear. In a few discharges, however, transition-like behavior is observed. Figure 5 shows a discharge with higher neutral beam injection power $(5 \mathrm{MW})$. At $0.45 \mathrm{sec}$ after the limiter bias turn-on, transitionlike behavior similar to Fig. 4 is observed. After the transition, $\mathrm{H}_{\alpha}$ emis sion was reduced both at the limiter and at the wall. Impurity levels and radiation power increased significantly, which made the discharge unstable. 


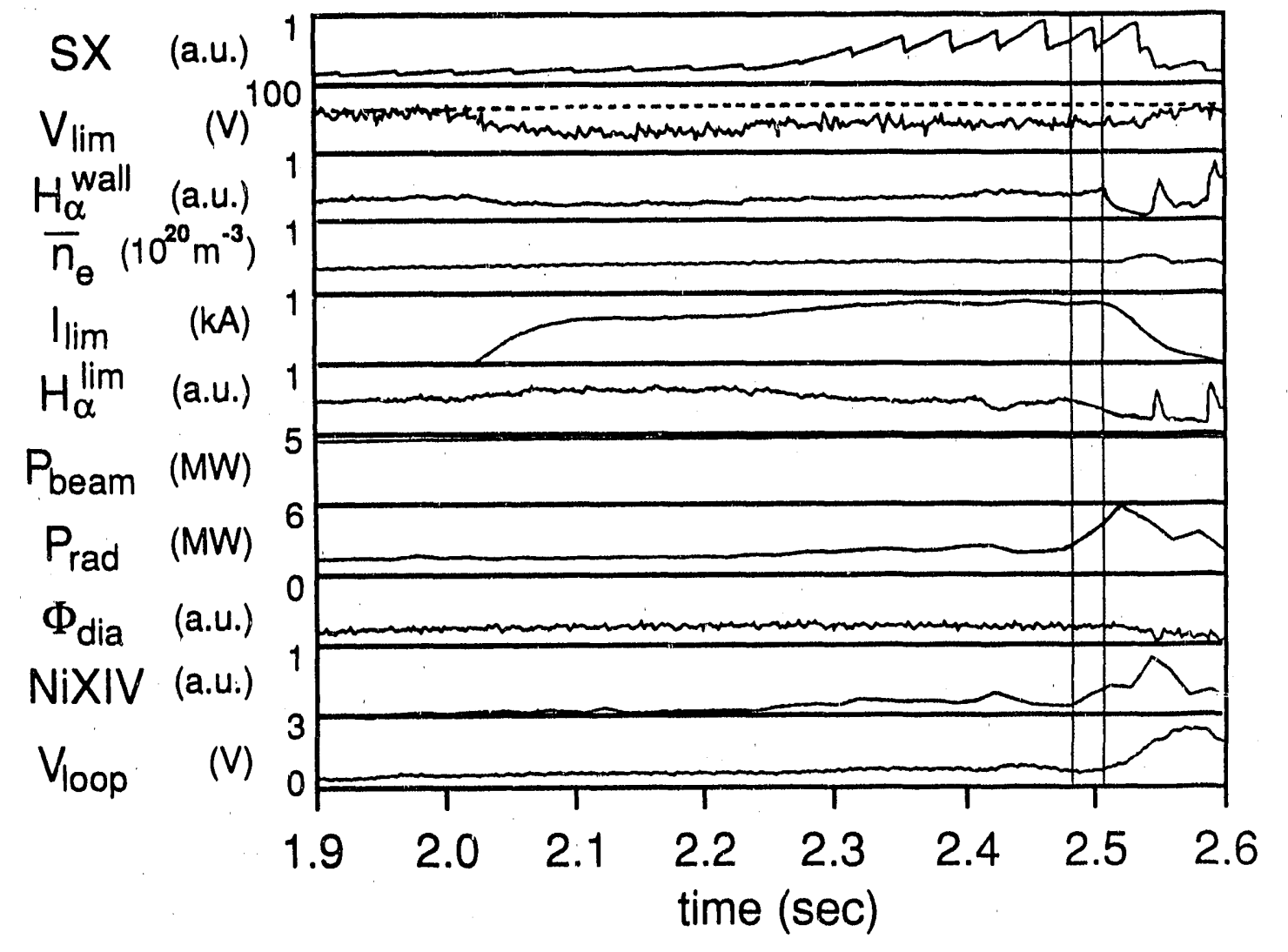

Fig. 5. Negative divertor bias with $5 \mathrm{MW}$ neutral beam heating. 


\section{DISCUSSION}

The particle recycling characteristics exhibited with small, negative limiter voltages were similar to the ones observed in MACROTOR, PBX, and TEXTOR. As limiter voltage was negative with respect to the wall, the ions tend to flow toward the limiter and the electrons flow toward the wall. This can be understood as transport process in the scrape-off layer. However, the change in edge Bremsstrahlung emission $(r / a \approx 0.8)$ with both bias polarities, and reduction in particle recycling both at the limiter and the wall cannot be understood as the process just in the scrape-off layer. These phenomena can be only understood as a change in transport process inside the limiter-defined surface. As the potential is applied at the limiter, radial electric field near the limiter-defined surface becomes negative, edge plasma starts to rotate, and the radial electric field penetrates inside the limiter defined surface via momentum transfer by radial viscosity or charge-exchange process. The reason for the bifurcation-like behavior is not clear yet. The increase in impurity levels can be due to either improvement in confinement, or increase in impurity influx, or both. As the particle confinement is improved, it may be natural to suppose that impurity confinement is improved as well. If the improvement in particle confinement is due to negative electric field at the edge, impurities should be confined better than the particles, since impurities feel stronger electric force due to their multiple charges.

In this investigation, the effects of edge radial electric field on energy confinement is not clear due to increase in radiation loss power. In DIII-D and JFT-2M, two rows of divertor plates will be biased with respect to the wall and with respect to each other to investigate edge potential and edge current in particle and energy confinement, stability, current drive, and impurity control $[10,11]$. In these experiments, impurity 
control by divertor would allow us to do a conclusive investigation on the role of edge electric field in energy confinement. 


\section{SUMMARY}

Experiments in DIII-D demonstrated following characteristics with an electrically bidsed limiter.

1. $H_{\alpha}$ signals both at the limiter and at the inner wall were reduced by a factor of 2.3 by strong negative biasing $\left(V_{\lim } \approx-300 \mathrm{~V}\right)$. In these discharges, the line-average electron densities were approxinately the same with and without limiter bias, which suggests that $\tau_{\mathrm{p}}$ was improved by negative bias.

2. Edge 13remsstrahlung emission signals were reduced by a factor of two. The central chord Bremsstrahlung signal did not change with bias, suggesting that the electron density profile has a steep gradient at the plasma edge.

3. Repetitive bursts were observed in $\mathrm{H}_{\alpha}$ signals and edge Bremsstrahlung signals.

4. Impurity leveis increased with limiter bias, which induced major disruptions.

5. No improvements of $\tau_{E}$ was observed.

These experimental data suggest that particle confinement characteristics of limiter biased discharges (1-4) are similar to those with divertor $\mathrm{H}$-mode. We have not observed improvement of $\tau_{E}$, which might simply imply that the convection loss is not an important energy transport mechanism with the plasma parameter range investigated. Increased level of impurities and radiation loss power could also have prevented the improvement of energy confinement. In high density discharges and neutral beam heated discharges, the global energy confinement could be dominated by edge convection loss. In those discharges the global energy confinement might be 
improved by the limiter bias. In addition, the impurity level would be suppressed in high density discharges. 


\section{ACKNOWLEDGMENTS}

The authors would like to appreciate fruitful discussions and continuous encouragement by Drs. R.D. Stambaugh, J. Luxon, D. Overskei, T. Ohkawa, and T. Simonen. One of the authors (M.S.) would like to express sincere thanks to Drs. S. Konoshima, S. Tamura, and M. Yoshikawa for continuous support.

This work was supported by the U.S. Department of Energy under Contract No. DE-AC03-89ER51114. 


\section{REFERENCES}

[1] Burrell, K.H., etal., Plasma Phys. and Controlled Nucl. Fusion 31 (1989) 1649.

[2] Ida, K., private communication.

[3] Shimada, M., etal., in Plasma Physics and Controlled Nuclear Fusion Research (Proc. 10th Int. Conf. London, 1984) Vol. I, IAEA, Vienna, 281.

[4] Oren, L., etal., J. Nucl. Mat. 111 \& 112 (1982) 34.

[5] Shimada, M., etal., Bull. Amer. Phys. Soc. 30 (1985) 1439.

[6] Phillips, P.E., etal., J. Nucl. Mat. 145 \& 147 (1987) 807.

[7] Conn, R., etal., in Plasma Physics and Controlled $\mathrm{N}_{1}$ lear Fusion Research (Proc. 11th Int. Conf. Kyoto, 1986) Vol. I, IAEA Vienna, 249.

[8] Taylor, R., et al., Phys. Rev. Lett. 63 (1989) 2365.

[9] Luxon, J., etal., in Plasma Physics and Controlled Nuclear Fusion Research (Proc. 11th Int. Conf. Kyoto, 1986) Vol. I, IAEA, Vienna, 159.

[10] Stambaugh, R.D., etal., Bull. Amer. Phys. Soc. 31 (1986) 1535.

[11] Shimada, M., etal., in Proc. IAEA Technical Committee Meeting on Impurity Control (Naka, February 1989), IAEA-TECDOC-536, IAEA, Vienna (1990) 31. 

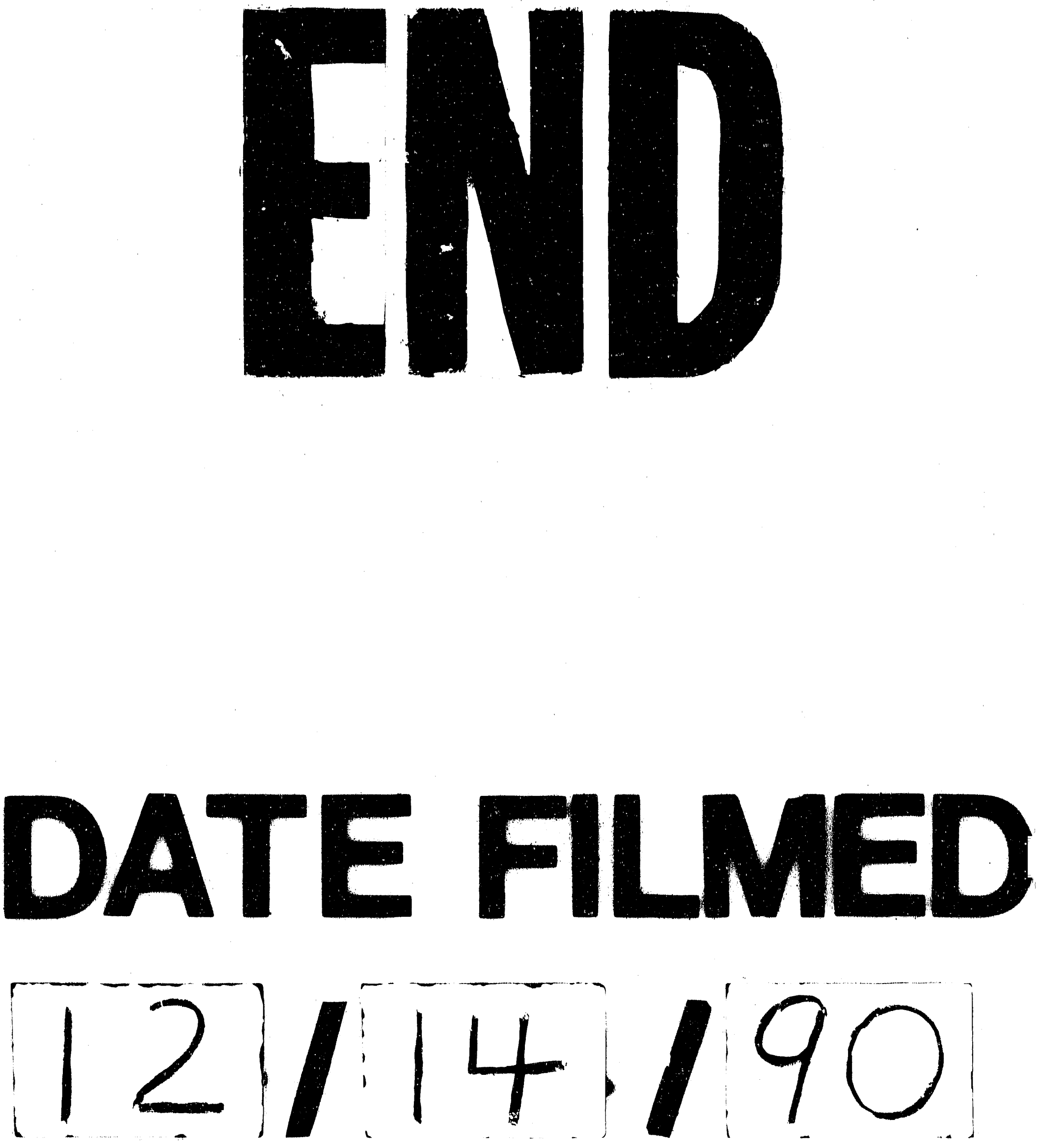
